\title{
Discrete Maximum Principle and Energy Stability of Compact Difference Scheme for the Allen-Cahn Equation
}

\author{
Dan $\operatorname{Tian}^{1}$, Yuanfeng Jin ${ }^{1 \dagger}$ and Gang $\mathrm{Lu}^{2}$ \\ 1 Department of Mathematics, Yanbian University, Yanji 133001, P.R. China; 2 Department of Mathematics, \\ School of Science, ShenYang University of Technology, Shenyang 110870, P.R. China (email: \\ 1060208723@qq.com, yfkim@ybu.edu.cn, lvgang1234@hanmail.net)
}

\begin{abstract}
In the paper, a fully discrete compact difference scheme with $O\left(\tau^{2}+h^{4}\right)$ precision is established by considering the numerical approximation of the one-dimensional Allen-Cahn equation. The numerical solutions satisfy discrete maximum principle under reasonable step ratio and time step constraint is proved. And the energy stability for the fully discrete scheme is investigated. An example is finally presented to show the effectiveness of scheme.
\end{abstract}

Key words: Allen-Cahn Equation; Compact Difference Scheme; Maximum Principle; Energy Stability.

\section{Introduction}

The phase field model is a mathematical model which is described by partial differential equations. Due to its complexity, the numerical simulation of phase field are all along the very important problem which has been discussed at home and abroad, there are some theory and realistic sense for us to deal with the problems. In 1979, Allen and Cahn introduced the Allen-Cahn equation to describe the anti-phase boundary motion in the crystal. This equation describes fluid dynamics problems and reaction diffusion problems in materials science, and also proposes the same model in the study of many diffusion phenomena such as competition and rejection of biological populations, migration processes of riverbeds. For describing the anti-phase boundary motion in a crystal, since there is no exact solution for such a phase field model, the model was simulated by numerical method. At present numerical approximation methods for such phase field models include finite difference methods, finite element methods $[8,9]$, and spectral methods [6].

This paper presents the compact difference method to approximate the one-dimensional nonlinear Allen-Cahn equation with initial boundary conditions.

$$
\begin{cases}\frac{\partial u}{\partial t}=\varepsilon^{2} \Delta u-f(u), & x \in \Omega, t \in[0, T] . \\ u(x, 0)=u_{0}(x), & x \in \bar{\Omega} . \\ \left.u\right|_{\partial \Omega}=0, & t \in[0, T] .\end{cases}
$$

where $u$ indicates the concentration of a metal part in a binary alloy, the parameter $\varepsilon>0$ is the width of the interface, and the nonlinear term $f(u)=u^{3}-u$.

Define the energy function in $L^{2}$ - space

$$
E(u)=\oint_{\Omega}\left(F(u)-\frac{1}{2} \varepsilon^{2} u \triangle u\right) d x .
$$


where $F(u)=\frac{1}{4}\left(u^{2}-1\right)^{2}$. One of the intrinsic properties of the Allen-Cahn equation is that the energy function is decreasing with time,

$$
\frac{d}{d t} E(u) \leq 0, \quad \forall t>0 .
$$

It is known that the Allen-Cahn equation satisfies the maximum principle and energy stability. Then is it true that these properties are true for numerical approximation solutions? In 2010, Shen Jie established first-order semi-implicit scheme, second-order semi-implicit scheme and secondorder implicit scheme for Allen-Cahn equation and Cahn-Hilliard equation, and completed energy digressive stability analysis and error analysis [10]. In 2016, Zhang Jiaqi and Hou Tianliang considered three discrete schemes of the Allen-Cahn equation: stable first-order linear implicit-explicit scheme, stable second-order nonlinear correction Crank-Nicolson scheme and stable second-order linear LeapFrog scheme, proved that these three schemes satisfy the discrete maximum principle and energy stability [1]; Tang Tao proved the maximum principle and the unconditional stability of the firstorder implicit-explicit scheme of the Allen-Cahn equation, and gave an error analysis [2]. In 2017, Zheng Nan gave two high-efficiency numerical schemes for solving the Allen-Cahn equation, compared the operational efficiencies of the two numerical solutions, and verified the energy diminishing law by numerical examples [3]; Hou Tian Liang and Tang Tao established the second-order CrankNicolson scheme in time and second-order central difference approximation in space for fractional-inspace Allen-Cahn equation, which proved that the numerical solution satisfies the discrete maximum principle and energy stability under reasonable time step constraints, and gave an error analysis [5].

Based on the existing finite difference methods, a discrete format with four-order precision-the compact difference scheme, is established to perform related numerical analysis. The main research is two aspects: one is the discrete maximum principle: the numerical solutions for (1.1) can be bounded by 1 under the condition that the initial data is bounded by 1 ; the other is the energy stability: the defined discrete energy is decremented.

\section{Establishment of the Compact Difference Scheme}

In this section, the compact difference scheme with $O\left(\tau^{2}+h^{4}\right)$ precision is established for the one-dimensional Allen-Cahn equation (1.1) .

First, three common numerical differential formulas were described.

Let $h>0$ and $c$ be two constants,

(1)If $g(x) \in C^{2}[c-h, c+h]$, there is

$$
g(c)=\frac{1}{2}[g(c-h)+g(c+h)]-\frac{h^{2}}{2} g^{\prime \prime}\left(\xi_{1}\right), \quad c-h<\xi_{1}<c+h .
$$

(2)If $g(x) \in C^{3}[c-h, c+h]$, there is

$$
g^{\prime}(c)=\frac{1}{2 h}[g(c+h)-g(c-h)]-\frac{h^{2}}{6} g^{\prime \prime \prime}\left(\xi_{2}\right), \quad c-h<\xi_{2}<c+h .
$$

(3)If $g(x) \in C^{6}[c-h, c+h]$, there is

$$
\begin{gathered}
\frac{1}{12}\left[g^{\prime \prime}(c-h)+10 g^{\prime \prime}(c)+g^{\prime \prime}(c+h)\right]=\frac{1}{h^{2}}[g(c+h)-2 g(c)+g(c-h)]+\frac{h^{4}}{240} g^{(6)}\left(\xi_{3}\right), \\
c-h<\xi_{3}<c+h .
\end{gathered}
$$


Next, we divide equally the solution interval $x \in \Omega, t \in[0, T]$ into $\mathrm{N}$ equal segments. Let $h=\frac{\Omega}{N}, \tau=\frac{T}{N}$, where $h$ and $\tau$ are the space step and time step. Denote $\Omega_{h \tau}=\left\{u_{i}^{n} \mid 0 \leq i \leq\right.$ $N, 0 \leq n \leq N\}$ is called grid function, introduce the following notations,

$$
\begin{array}{rlrl}
\delta_{t} u_{i}^{n+\frac{1}{2}} & =\frac{1}{\tau}\left(u_{i}^{n+1}-u_{i}^{n}\right) . & \delta_{x}^{2} u_{i}^{n}=\frac{1}{h^{2}}\left(u_{i+1}^{n}-2 u_{i}^{n}+u_{i-1}^{n}\right) . \\
u_{i}^{n+\frac{1}{2}}=\frac{1}{2}\left(u_{i}^{n+1}+u_{i}^{n}\right) . & \left(u_{i}^{n+\frac{1}{2}}\right)^{3}=\frac{1}{2}\left[\left(u_{i}^{n+1}\right)^{3}+\left(u_{i}^{n}\right)^{3}\right] .
\end{array}
$$

where

$$
u_{i}^{n}=u\left(x_{i}, t_{n}\right), \quad 0 \leq i \leq N, \quad 0 \leq n \leq N .
$$

Let $u=\left\{u_{i} \mid 0 \leq i \leq N\right\}$, define the operator

$$
(\mathcal{A} u)_{i}= \begin{cases}\frac{1}{12}\left(u_{i-1}+10 u_{i}+u_{i+1}\right), & 1 \leq i \leq N-1, \\ u_{i}, & i=0, N .\end{cases}
$$

Considering equation (1.1) at the point $\left(x_{i}, t_{n+\frac{1}{2}}\right)$, we obtain

$$
\frac{\partial u}{\partial t}\left(x_{i}, t_{n+\frac{1}{2}}\right)=\varepsilon^{2} \frac{\partial^{2} u}{\partial x^{2}}\left(x_{i}, t_{n+\frac{1}{2}}\right)-f\left(x_{i}, t_{n+\frac{1}{2}}\right), \quad 0 \leq i \leq N, \quad 0 \leq n \leq N-1 .
$$

Using formula (2.1) and (2.2), we have

$$
\begin{gathered}
\frac{\partial u}{\partial t}\left(x_{i}, t_{n+\frac{1}{2}}\right)=\frac{1}{\tau}\left[u\left(x_{i}, t_{n+1}\right)-u\left(x_{i}, t_{n}\right)\right]-\frac{\tau^{2}}{24} \cdot \frac{\partial^{3} u}{\partial t^{3}}\left(x_{i}, \theta_{i n}\right) . \\
\frac{\partial^{2} u}{\partial x^{2}}\left(x_{i}, t_{n+\frac{1}{2}}\right)=\frac{1}{2}\left[\frac{\partial^{2} u}{\partial x^{2}}\left(x_{i}, t_{n}\right)+\frac{\partial^{2} u}{\partial x^{2}}\left(x_{i}, t_{n+1}\right)\right]-\frac{\tau^{2}}{8} \cdot \frac{\partial^{4} u}{\partial x^{2} \partial t^{2}}\left(x_{i}, \widetilde{\theta}_{i n}\right) .
\end{gathered}
$$

where $\theta_{i n}, \widetilde{\theta}_{i n} \epsilon\left(t_{n}, t_{n+1}\right)$. Then we get

$$
\begin{aligned}
\delta_{t} u_{i}^{n+\frac{1}{2}}= & \varepsilon^{2} \cdot \frac{1}{2}\left[\frac{\partial^{2} u}{\partial x^{2}}\left(x_{i}, t_{n}\right)+\frac{\partial^{2} u}{\partial x^{2}}\left(x_{i}, t_{n+1}\right)\right]-f\left(x_{i}, t_{n+\frac{1}{2}}\right) \\
& +\left[\frac{1}{24} \cdot \frac{\partial^{3} u}{\partial t^{3}}\left(x_{i}, \theta_{i n}\right)-\frac{\varepsilon^{2}}{8} \cdot \frac{\partial^{2} v}{\partial t^{2}}\left(x_{i}, \widetilde{\theta}_{i n}\right)\right] \tau^{2}, \quad 0 \leq i \leq N, \quad 0 \leq n \leq N-1 .
\end{aligned}
$$

Acting operator $\mathcal{A}$ on both sides of the above formula, we obtain

$$
\begin{aligned}
\mathcal{A} \delta_{t} u_{i}^{n+\frac{1}{2}}= & \varepsilon^{2} \cdot \frac{1}{2}\left[\mathcal{A} \frac{\partial^{2} u}{\partial x^{2}}\left(x_{i}, t_{n}\right)+\mathcal{A} \frac{\partial^{2} u}{\partial x^{2}}\left(x_{i}, t_{n+1}\right)\right]-\mathcal{A} f\left(x_{i}, t_{n+\frac{1}{2}}\right) \\
& +\mathcal{A}\left[\frac{1}{24} \cdot \frac{\partial^{3} u}{\partial t^{3}}\left(x_{i}, \theta_{i n}\right)-\frac{\varepsilon^{2}}{8} \cdot \frac{\partial^{2} v}{\partial t^{2}}\left(x_{i}, \widetilde{\theta}_{i n}\right)\right] \tau^{2}, \quad 1 \leq i \leq N-1, \quad 0 \leq n \leq N-1 .
\end{aligned}
$$

Using formula (2.3), we have

$$
\mathcal{A} \frac{\partial^{2} u}{\partial x^{2}}\left(x_{i}, t_{n}\right)=\delta_{x}^{2} u_{i}^{n}+\frac{h^{4}}{240} \cdot \frac{\partial^{6} u}{\partial x^{6}}\left(\xi_{\text {in }}, t_{n}\right) .
$$

where $\xi_{i n} \epsilon\left(x_{i-1}, x_{i+1}\right)$. Add the two equations of (2.7) that labeled $n$ and $n+1$, and divide by 2 , we have

$$
\frac{1}{2}\left[\mathcal{A} \frac{\partial^{2} u}{\partial x^{2}}\left(x_{i}, t_{n}\right)+\mathcal{A} \frac{\partial^{2} u}{\partial x^{2}}\left(x_{i}, t_{n+1}\right)\right]=\frac{1}{2}\left(\delta_{x}^{2} u_{i}^{n}+\delta_{x}^{2} u_{i}^{n+1}\right)+\frac{h^{4}}{240} \cdot \frac{\partial^{6} u}{\partial x^{6}}\left(\bar{\xi}_{i n}, \bar{t}_{i n}\right) .
$$


where $\bar{\xi}_{i n} \epsilon\left(x_{i-1}, x_{i+1}\right) \bar{t}_{i n} \epsilon\left(t_{n}, t_{n+1}\right)$.

Substituting (2.8) into (2.7), we obtain

$$
\mathcal{A} \delta_{t} u_{i}^{n+\frac{1}{2}}=\varepsilon^{2} \frac{\left(\delta_{x}^{2} u_{i}^{n}+\delta_{x}^{2} u_{i}^{n+1}\right)}{2}-\mathcal{A} f\left(x_{i}, t_{n+\frac{1}{2}}\right)+R_{i n}, \quad 1 \leq i \leq N-1, \quad 0 \leq n \leq N-1 .
$$

where

$$
R_{i n}=\mathcal{A}\left[\frac{1}{24} \cdot \frac{\partial^{3} u}{\partial t^{3}}\left(x_{i}, \theta_{i n}\right)-\frac{\varepsilon^{2}}{8} \cdot \frac{\partial^{2} v}{\partial t^{2}}\left(x_{i}, \widetilde{\theta}_{i n}\right)\right] \tau^{2}+\frac{\varepsilon^{2}}{240} \cdot \frac{\partial^{6} u}{\partial x^{6}}\left(\bar{\xi}_{i n}, \bar{t}_{i n}\right) h^{4} .
$$

In (2.9), omit the error term $R_{\text {in }}$ and substitute the following

$$
f\left(x_{i}, t_{n+\frac{1}{2}}\right)=\frac{\left(u_{i}^{n+1}\right)^{3}+\left(u_{i}^{n}\right)^{3}}{2}-\frac{u_{i}^{n+1}+u_{i}^{n}}{2} .
$$

Then we can get the Allen-Cahn equation (1.1) corresponding compact difference scheme:

$$
\begin{array}{r}
\mathcal{A} \frac{u_{i}^{n+1}-u_{i}^{n}}{\tau}+\mathcal{A}\left[\frac{\left(u_{i}^{n+1}\right)^{3}+\left(u_{i}^{n}\right)^{3}}{2}-\frac{u_{i}^{n+1}+u_{i}^{n}}{2}\right]=\frac{\varepsilon^{2}}{2}\left(\delta_{x}^{2} u_{i}^{n+1}+\delta_{x}^{2} u_{i}^{n}\right), \\
1 \leq i \leq N-1, \quad 0 \leq n \leq N-1 .
\end{array}
$$

Finally, we use the second-order central finite difference to approximate the spatial derivatives and denote $D_{h}$ as the corresponding discrete matrix, so $D_{h}$ is given by

$$
D_{h}=\frac{1}{h^{2}}\left(\begin{array}{cccc}
-2 & 1 & 0 & 0 \\
1 & -2 & \ddots & 0 \\
0 & \ddots & \ddots & 1 \\
0 & 0 & 1 & -2
\end{array}\right)_{N \times N}
$$

where $N$ indicates the number of internal nodes in the space, and $h$ indicates the space step. It can be verified that the discrete matrix $D_{h}$ satisfies the following properties:

(1) $D_{h}$ is symmetric;

(2) $D_{h}$ is negative semidefinite, i.e.

$$
U^{T} D_{h} U \leq 0, \quad \forall U \in R^{N}
$$

(3)Elements of $D_{h}$ satisfy

$$
d_{i i}=-b<0, \quad b \geq \max _{1 \leq i \leq N} \sum_{j \neq i}\left|b_{i j}\right| .
$$

To represent the average operator, $\mathcal{A}$ can be expressed as:

$$
A=\left(\begin{array}{cccc}
\frac{10}{12} & \frac{1}{12} & 0 & 0 \\
\frac{1}{12} & \frac{10}{12} & \ddots & 0 \\
0 & \ddots & \ddots & \frac{1}{12} \\
0 & 0 & \frac{1}{12} & \frac{10}{12}
\end{array}\right)_{N \times N}
$$

It can be verified that the matrix $A$ satisfies the following properties:

(1) $A$ is symmetric; 
(2) $A$ is positive, i.e.

$$
U^{T} A U>0, \quad \forall U \in R^{N}
$$

(3)Elements of $A$ satisfy

$$
a_{i j}>0, \quad\|A\|_{\infty}=\max _{1 \leq i \leq N} \sum_{j=1}^{N} a_{i j}=1
$$

Substituting the matrix $D_{h}$ and $A$ into (2.11), we construct the compact difference scheme as follow:

$$
A \frac{U^{n+1}-U^{n}}{\tau}+A\left[\frac{\left(U^{n+1}\right)^{\cdot 3}-U^{n+1}}{2}+\frac{\left(U^{n}\right)^{\cdot 3}-U^{n}}{2}\right]=\frac{\varepsilon^{2}\left(D_{h} U^{n+1}+D_{h} U^{n}\right)}{2}
$$

where

$$
\begin{aligned}
& U^{n}:=\left(U_{1}^{n}, U_{2}^{n}, \cdots, U_{N}^{n}\right)^{T}, \\
& \left(U^{n}\right)^{3}:=\left(\left(U_{1}^{n}\right)^{3},\left(U_{2}^{n}\right)^{3}, \cdots,\left(U_{N}^{n}\right)^{3}\right)^{T} .
\end{aligned}
$$

On both sides of the compact difference scheme (2.18) multiplied by matrix $A^{-1}$, we obtain,

$$
\frac{U^{n+1}-U^{n}}{\tau}+\left[\frac{\left(U^{n+1}\right)^{3}-U^{n+1}}{2}+\frac{\left(U^{n}\right)^{\cdot 3}-U^{n}}{2}\right]=\frac{\varepsilon^{2}}{2} A^{-1} D_{h}\left(U^{n+1}+U^{n}\right) .
$$

It can be verified that the matrix $A^{-1} D_{h}$ satisfies the following properties:

(1) $A^{-1} D_{h}$ is symmetric;

(2) $A^{-1} D_{h}$ is negative, i.e.

$$
U^{T} A^{-1} D_{h} U \leq 0, \quad \forall U \in R^{N}
$$

\section{The Discrete Maximum Principle}

Theorem 3.1. Let us consider the Allen-Cahn problem (1.1). If the initial value is bounded by 1 , i.e. $\max _{x \in \bar{\Omega}}\left|u_{0}(x)\right| \leq 1$, then the numerical solution of the fully discrete scheme (2.18) is also bounded by 1 in the sense that $\left\|U^{n}\right\|_{\infty} \leq 1$ for all $n \geq 1$, and the step ratio satisfies $\frac{1}{6} \leq \lambda \leq \frac{5}{6}$, and the time step size satisfies $0<\tau \leq \min \left\{\lambda-\frac{1}{6}, 1-\frac{6}{5} \lambda\right\}$.

Proof. Obviously, $\left\|U^{0}\right\| \infty \leq\left\|u_{0}\right\| \leq 1$. We assume $\left\|U^{n}\right\| \infty \leq 1$ and will verify the result is true for $\left\|U^{n+1}\right\| \infty \leq 1$.

Expand the established compact difference scheme (2.11),

$$
\begin{array}{r}
\frac{1}{12}\left[\frac{u_{i-1}^{n+1}-u_{i-1}^{n}}{\tau}+\frac{\left(u_{i-1}^{n+1}\right)^{3}+\left(u_{i-1}^{n}\right)^{3}}{2}-\frac{u_{i-1}^{n+1}+u_{i-1}^{n}}{2}\right]+\frac{10}{12}\left[\frac{u_{i}^{n+1}-u_{i}^{n}}{\tau}+\frac{\left(u_{i}^{n+1}\right)^{3}+\left(u_{i}^{n}\right)^{3}}{2}-\frac{u_{i}^{n+1}+u_{i}^{n}}{2}\right] \\
+\frac{1}{12}\left[\frac{u_{i+1}^{n+1}-u_{i+1}^{n}}{\tau}+\frac{\left(u_{i+1}^{n+1}\right)^{3}+\left(u_{i+1}^{n}\right)^{3}}{2}-\frac{u_{i+1}^{n+1}+u_{i+1}^{n}}{2}\right]=\frac{\varepsilon^{2}}{2}\left(\frac{u_{i-1}^{n+1}-2 u_{i}^{n+1}+u_{i+1}^{n+1}}{h^{2}}+\frac{u_{i-1}^{n}-2 u_{i}^{n}+u_{i+1}^{n}}{h^{2}}\right) .
\end{array}
$$

Let $\lambda=\varepsilon^{2} \frac{\tau}{h^{2}}$ on both sides of the above formula multiplied by $\tau$, and shift item,gives

$$
\frac{10}{12}\left[u_{i}^{n+1}+\frac{\tau}{2}\left(u_{i}^{n+1}\right)^{3}-\frac{\tau}{2} u_{i}^{n+1}\right]+\frac{1}{12}\left[u_{i-1}^{n+1}+\frac{\tau}{2}\left(u_{i-1}^{n+1}\right)^{3}-\frac{\tau}{2} u_{i-1}^{n+1}\right]
$$




$$
\begin{aligned}
& +\frac{1}{12}\left[u_{i+1}^{n+1}+\frac{\tau}{2}\left(u_{i+1}^{n+1}\right)^{3}-\frac{\tau}{2} u_{i+1}^{n+1}\right]-\frac{\lambda}{2}\left(u_{i-1}^{n+1}-2 u_{i}^{n+1}+u_{i+1}^{n+1}\right) \\
= & \frac{10}{12}\left[u_{i}^{n}-\frac{\tau}{2}\left(u_{i}^{n}\right)^{3}+\frac{\tau}{2} u_{i}^{n}\right]+\frac{1}{12}\left[u_{i-1}^{n}-\frac{\tau}{2}\left(u_{i-1}^{n}\right)^{3}+\frac{\tau}{2} u_{i-1}^{n}\right] \\
& +\frac{1}{12}\left[u_{i+1}^{n}-\frac{\tau}{2}\left(u_{i+1}^{n}\right)^{3}+\frac{\tau}{2} u_{i+1}^{n}\right]+\frac{\lambda}{2}\left(u_{i-1}^{n}-2 u_{i}^{n}+u_{i+1}^{n}\right) .
\end{aligned}
$$

We obtain

$$
\begin{aligned}
{\left[\frac{10}{12}\left(1-\frac{\tau}{2}\right)+\lambda\right] u_{i}^{n+1}+\frac{10}{12} \cdot \frac{\tau}{2}\left(u_{i}^{n+1}\right)^{3}=} & {\left[\frac{\lambda}{2}-\frac{1}{12}\left(1-\frac{\tau}{2}\right)\right] u_{i-1}^{n+1}-\frac{1}{12} \cdot \frac{\tau}{2}\left(u_{i-1}^{n+1}\right)^{3} } \\
& +\left[\frac{\lambda}{2}-\frac{1}{12}\left(1-\frac{\tau}{2}\right)\right] u_{i+1}^{n+1}-\frac{1}{12} \cdot \frac{\tau}{2}\left(u_{i+1}^{n+1}\right)^{3} \\
& +\left[\frac{\lambda}{2}+\frac{1}{12}\left(1+\frac{\tau}{2}\right)\right] u_{i-1}^{n}-\frac{1}{12} \cdot \frac{\tau}{2}\left(u_{i-1}^{n}\right)^{3} \\
& +\left[\frac{10}{12}\left(1+\frac{\tau}{2}\right)-\lambda\right] u_{i}^{n}-\frac{10}{12} \cdot \frac{\tau}{2}\left(u_{i}^{n}\right)^{3} \\
& +\left[\frac{\lambda}{2}+\frac{1}{12}\left(1+\frac{\tau}{2}\right)\right] u_{i+1}^{n}-\frac{1}{12} \cdot \frac{\tau}{2}\left(u_{i+1}^{n}\right)^{3} .
\end{aligned}
$$

For the right side of (3.1), the sum of the last three items is recorded as $P$.

Let $\left\|U^{n}\right\|_{\infty}=x$, we assume $\left\|U^{n}\right\|_{\infty} \leq 1$.

Let

$$
f(x)=\left[\frac{10}{12}\left(1+\frac{\tau}{2}\right)-\lambda\right] x-\frac{10}{12} \cdot \frac{\tau}{2} x^{3}, \quad x \in[0,1] .
$$

This gives that

$$
f(0)=0, \quad f(1)=\frac{5}{6}-\lambda
$$

and

$$
\begin{aligned}
& f^{\prime}(x)=\frac{5}{6}\left(1+\frac{\tau}{2}\right)-\lambda-\frac{5 \tau}{4} x^{2} . \\
& f^{\prime \prime}(x)=-\frac{5 \tau}{2} x \leq 0 .
\end{aligned}
$$

So, we can get $f^{\prime}(x)$ is decreasing,

$$
f^{\prime}(0)=\frac{5}{6}\left(1+\frac{\tau}{2}\right)-\lambda, \quad f^{\prime}(1)=\frac{5}{6}\left(1+\frac{\tau}{2}\right)-\lambda-\frac{5 \tau}{4} .
$$

It can be verified that if $\tau \leq 1-\frac{6}{5} \lambda$ then $f^{\prime}(1) \geq 0$, we have $f^{\prime}(x) \geq 0$. Therefore, $f(x) \leq f(1)$ for all $x \in[0,1]$, gives

$$
f(x) \leq \frac{5}{6}-\lambda, \quad \lambda \leq \frac{5}{6}
$$

Let

$$
g(x)=\left[\frac{\lambda}{2}+\frac{1}{12}\left(1+\frac{\tau}{2}\right)\right] x-\frac{1}{12} \cdot \frac{\tau}{2} x^{3}, \quad x \in[0,1] .
$$

Similarly, we have

$$
g(x) \leq \frac{\lambda}{2}+\frac{1}{12}
$$


together with (3.2) and (3.3), we obtain

$$
P \leq f(1)+2 g(1)=1
$$

For the first two terms to the right side of $(3.1)$, denote $U^{n+1}=y$. Let

$$
\begin{gathered}
h(y)=\left[\frac{\lambda}{2}-\frac{1}{12}\left(1-\frac{\tau}{2}\right)\right] y-\frac{1}{12} \cdot \frac{\tau}{2} y^{3} . \\
h(0)=0, \quad h(1)=\frac{\lambda}{2}-\frac{1}{12} .
\end{gathered}
$$

We have

$$
h^{\prime}(y)=\frac{\lambda}{2}-\frac{1}{12}\left(1-\frac{\tau}{2}\right)-\frac{\tau}{8} y^{2}
$$

When $h^{\prime}(y)=0$, find the maximum point $y_{0}=\sqrt{\frac{8}{\tau}} \cdot \sqrt{\frac{1}{2}\left(\lambda-\frac{1}{6}\right)+\frac{\tau}{24}}$, where $\lambda \geq \frac{1}{6}$.

Since $h(y)$ is an odd function, it is increasing in $\left(-y_{0}, y_{0}\right)$, gives $|h(y)| \leq\left|h\left(y_{0}\right)\right|$.

Substituting (3.4) and (3.5) into (3.1), we can obtain

$$
\left[\frac{10}{12}\left(1-\frac{\tau}{2}\right)+\lambda\right] u_{i}^{n+1}+\frac{10}{12} \cdot \frac{\tau}{2}\left(u_{i}^{n+1}\right)^{3} \leq h\left(u_{i-1}^{n+1}\right)+h\left(u_{i+1}^{n+1}\right)+1, \quad 1 \leq i \leq N-1 .
$$

Assume $\left\|U^{n+1}\right\|_{\infty}=\left|U_{i_{0}}^{n+1}\right|=M$.

On the one hand, for both $h\left(u_{i-1}^{n+1}\right)$ and $h\left(u_{i+1}^{n+1}\right)$, the absolute value is taken on both sides of the equation. According to the triangle inequality, we have

$$
\begin{aligned}
\left|h\left(u_{i-1}^{n+1}\right)\right| & \left.=\mid \frac{\lambda}{2}-\frac{1}{12}\left(1-\frac{\tau}{2}\right)\right] u_{i-1}^{n+1}-\frac{\tau}{24}\left(u_{i-1}^{n+1}\right)^{3} \mid \\
& \leq\left[\frac{\lambda}{2}-\frac{1}{12}\left(1-\frac{\tau}{2}\right)\right]\left|u_{i-1}^{n+1}\right|+\frac{\tau}{24}\left|u_{i-1}^{n+1}\right|^{3} \\
& \leq\left[\frac{\lambda}{2}-\frac{1}{12}\left(1-\frac{\tau}{2}\right)\right] M+\frac{\tau}{24} M^{3} . \\
\left|h\left(u_{i+1}^{n+1}\right)\right| & \left.=\mid \frac{\lambda}{2}-\frac{1}{12}\left(1-\frac{\tau}{2}\right)\right] u_{i+1}^{n+1}-\frac{\tau}{24}\left(u_{i+1}^{n+1}\right)^{3} \mid \\
& \leq\left[\frac{\lambda}{2}-\frac{1}{12}\left(1-\frac{\tau}{2}\right)\right]\left|u_{i+1}^{n+1}\right|+\frac{\tau}{24}\left|u_{i+1}^{n+1}\right|^{3} \\
& \leq\left[\frac{\lambda}{2}-\frac{1}{12}\left(1-\frac{\tau}{2}\right)\right] M+\frac{\tau}{24} M^{3} .
\end{aligned}
$$

Let the left side of (3.6) take $i=i_{0}$, then put (3.7) and (3.8) into the right sidegives

$$
\left[\lambda+\frac{5}{6}\left(1-\frac{\tau}{2}\right)\right] M+\frac{5 \tau}{12} M^{3} \leq\left[\lambda-\frac{1}{6}\left(1-\frac{\tau}{2}\right)\right] M+\frac{\tau}{12} M^{3}+1 .
$$

which is

$$
\left(1-\frac{\tau}{2}\right) M+\frac{\tau}{3} M^{3} \leq 1
$$

It is easy to verify that $M \leq \frac{1}{1-\frac{\tau}{2}} \leq 2$, and $\left|u_{i_{0}}^{n+1}\right| \in(0,2)$, where $\tau \leq 1$.

Suppose $y_{0}=2$, we can get $h(y)$ is monotonically increasing on $(-2,2)$,

$$
h^{\prime}(y)=\frac{\lambda}{2}-\frac{1}{12}\left(1-\frac{\tau}{2}\right)-\frac{\tau}{8} y^{2} \geq 0
$$




$$
\frac{\tau}{8} y^{2} \leq \frac{\lambda}{2}-\frac{1}{12}\left(1-\frac{\tau}{2}\right)=\frac{1}{2}\left(\lambda-\frac{1}{6}\right)+\frac{\tau}{24}
$$

Since $y \epsilon(-2,2)$,

$$
\frac{\tau}{2} \leq \frac{1}{2}\left(\lambda-\frac{1}{6}\right)
$$

This gives that

$$
\tau \leq \lambda-\frac{1}{6}
$$

On the other hand, take $i=i_{0}$ on the left side and $i-1=i_{0}, i+1=i_{0}$ on the right side of (3.6), we have

$$
\left[\lambda+\frac{5}{6}\left(1-\frac{\tau}{2}\right)\right]\left|u_{i_{0}}^{n+1}\right|+\frac{5 \tau}{12}\left(\left|u_{i_{0}}^{n+1}\right|\right)^{3} \leq h\left(\left|u_{i_{0}}^{n+1}\right|\right)+h\left(\left|u_{i_{0}}^{n+1}\right|\right)+1 .
$$

Using (3.5) gives

$$
\begin{gathered}
{\left[\lambda+\frac{5}{6}\left(1-\frac{\tau}{2}\right)\right] M+\frac{5 \tau}{12} M^{3} \leq\left[\lambda-\frac{1}{6}\left(1-\frac{\tau}{2}\right)\right] M-\frac{\tau}{12} M^{3}+1 .} \\
\left(1-\frac{\tau}{2}\right) M+\frac{\tau}{2} M^{3} \leq 1 .
\end{gathered}
$$

which yields $M \leq 1$.

In summary, when step ratio and time step satisfies $0<\tau \leq \min \left\{\lambda-\frac{1}{6}, 1-\frac{6}{5} \lambda\right\}, \quad \frac{1}{6} \leq \lambda \leq \frac{5}{6}$, we can conclude that $\left\|U^{n+1}\right\|_{\infty} \leq 1$.

This completes the proof of Theorem 1 .

\section{Stability of the Discrete Energy}

From the given function (1.2), the discrete energy function of the compact difference scheme (2.18) can be expressed as

$$
E(u)=\frac{1}{4} \cdot h \cdot \sum_{i=1}^{N}\left(U_{i}^{2}-1\right)^{2}-\frac{\varepsilon^{2}}{2} \cdot h \cdot U^{T} A^{-1} D_{h} U .
$$

Theorem 4.1. Consider the Allen-Cahn problem (1.1). If the initial value is bounded by 1, i.e. $\max \left|u_{0}(x)\right| \leq 1$, under the conditions in theorem (3.1), then the numerical solutions is obtained by

the scheme (2.18) satisfies the discrete energy decreasing property:

$$
E\left(U^{n+1}\right) \leq E\left(U^{n}\right) .
$$

Proof. Taking the difference of the discrete energy between two successive time levels, we get

$$
\begin{aligned}
\frac{E\left(U^{n+1}\right)-E\left(U^{n}\right)}{h}= & \frac{1}{4} \sum_{i=1}^{N}\left[\left(\left(U_{i}^{n+1}\right)^{2}-1\right)^{2}-\left(\left(U_{i}^{n}\right)^{2}-1\right)^{2}\right] \\
& -\frac{\varepsilon^{2}}{2}\left(\left(U^{n+1}\right)^{T} A^{-1} D_{h} U^{n+1}-\left(U^{n}\right)^{T} A^{-1} D_{h} U^{n}\right) .
\end{aligned}
$$


Note that following two fundamental inequalities hold for any $a, b \epsilon[-1,1]$

$$
\begin{aligned}
\left(a^{3}-a\right)(a-b)+(a-b)^{2} & \geq \frac{1}{4}\left[\left(a^{2}-1\right)^{2}-\left(b^{2}-1\right)^{2}\right] . \\
\left(a^{3}-b\right)(a-b)+(a-b)^{2} & \geq \frac{1}{4}\left[\left(a^{2}-1\right)^{2}-\left(b^{2}-1\right)^{2}\right] .
\end{aligned}
$$

Under the conditions in theorem (3.1), we have $\left\|U^{n}\right\|_{\infty} \leq 1$ and $\left\|U^{n+1}\right\|_{\infty} \leq 1$. Consequently, it follows from (4.4) and (4.5) that

$$
\begin{aligned}
& \frac{1}{4} \sum_{i=1}^{N}\left[\left(\left(U_{i}^{n+1}\right)^{2}-1\right)^{2}-\left(\left(U_{i}^{n}\right)^{2}-1\right)^{2}\right] \\
& \leq \sum_{i=1}^{N}\left[\frac{1}{2}\left(\left(U_{i}^{n+1}\right)^{3}-U_{i}^{n+1}\right)\left(U_{i}^{n+1}-U_{i}^{n}\right)+\frac{1}{2}\left(\left(U_{i}^{n}\right)^{3}-U_{i}^{n}\right)\left(U_{i}^{n+1}-U_{i}^{n}\right)+\left(U_{i}^{n+1}-U_{i}^{n}\right)^{2}\right]
\end{aligned}
$$

Since the matrix $A^{-1} D_{h}$ is symmetric, we have

$$
\frac{\varepsilon^{2}}{2}\left(U^{n+1}-U^{n}\right)^{T} A^{-1} D_{h}\left(U^{n+1}+U^{n}\right)=\frac{\varepsilon^{2}}{2}\left(\left(U^{n+1}\right)^{T} A^{-1} D_{h} U^{n+1}-\left(U^{n}\right)^{T} A^{-1} D_{h} U^{n}\right) .
$$

Combining (4.3), (4.6) and (4.7) get

$$
\begin{aligned}
& \frac{E\left(U^{n+1}\right)-E\left(U^{n}\right)}{h} \\
& \leq \sum_{i=1}^{N}\left[\frac{1}{2}\left(\left(U_{i}^{n+1}\right)^{3}-U_{i}^{n+1}\right)\left(U_{i}^{n+1}-U_{i}^{n}\right)+\frac{1}{2}\left(\left(U_{i}^{n}\right)^{3}-U_{i}^{n}\right)\left(U_{i}^{n+1}-U_{i}^{n}\right)+\left(U_{i}^{n+1}-U_{i}^{n}\right)^{2}\right] \\
& \quad-\frac{\varepsilon^{2}}{2}\left(U^{n+1}-U^{n}\right)^{T} A^{-1} D_{h}\left(U^{n+1}+U^{n}\right) .
\end{aligned}
$$

Taking $L^{2}$ inner product of (2.19) with $\left(U^{n+1}-U^{n}\right)$ obtain

$$
\begin{aligned}
& \sum_{i=1}^{N}\left[\frac{1}{2}\left(\left(U_{i}^{n+1}\right)^{3}-U_{i}^{n+1}\right)\left(U_{i}^{n+1}-U_{i}^{n}\right)+\frac{1}{2}\left(\left(U_{i}^{n}\right)^{3}-U_{i}^{n}\right)\left(U_{i}^{n+1}-U_{i}^{n}\right)+\frac{1}{\tau}\left(U_{i}^{n+1}-U_{i}^{n}\right)^{2}\right] \\
& =\frac{\varepsilon^{2}}{2}\left(U^{n+1}-U^{n}\right)^{T} A^{-1} D_{h}\left(U^{n+1}+U^{n}\right) .
\end{aligned}
$$

Consequently, together with (4.8) and (4.9), yields

$$
\begin{aligned}
& \frac{E\left(U^{n+1}\right)-E\left(U^{n}\right)}{h} \\
& \leq \sum_{i=1}^{N}\left[\frac{1}{2}\left(\left(U_{i}^{n+1}\right)^{3}-U_{i}^{n+1}\right)\left(U_{i}^{n+1}-U_{i}^{n}\right)+\frac{1}{2}\left(\left(U_{i}^{n}\right)^{3}-U_{i}^{n}\right)\left(U_{i}^{n+1}-U_{i}^{n}\right)+\left(U_{i}^{n+1}-U_{i}^{n}\right)^{2}\right] \\
& \quad-\sum_{i=1}^{N}\left[\frac{1}{2}\left(\left(U_{i}^{n+1}\right)^{3}-U_{i}^{n+1}\right)\left(U_{i}^{n+1}-U_{i}^{n}\right)+\frac{1}{2}\left(\left(U_{i}^{n}\right)^{3}-U_{i}^{n}\right)\left(U_{i}^{n+1}-U_{i}^{n}\right)+\frac{1}{\tau}\left(U_{i}^{n+1}-U_{i}^{n}\right)^{2}\right] .
\end{aligned}
$$

that is

$$
\frac{E\left(U^{n+1}\right)-E\left(U^{n}\right)}{h} \leq\left(1-\frac{1}{\tau}\right) \sum_{i=1}^{N}\left(U_{i}^{n+1}-U_{i}^{n}\right)^{2} .
$$

Since $0<\tau \leq \min \left\{\lambda-\frac{1}{6}, 1-\frac{6}{5} \lambda\right\}$, the right-hand side of (4.10) is non-positive, and hence (4.2) follows. 


\section{$5 \quad$ Numerical Tests}

In this section, some numerical examples are provided to validate the effectiveness on the energy stability and numerical maximum principle. We consider one-dimensional problems for (1.1) with homogeneous Neumann boundary condition. The initial condition is chose as

$$
u_{0}(x)=0.9 * \operatorname{rand}(\cdot)+0.05
$$

where " $\operatorname{rand}(\cdot)$ " represents a random number on each point in $[0,1]$.

First, we let the parameter $\epsilon^{2}=0.001$, and the mesh size in space is $h=0.01$. Denote $\lambda=\varepsilon^{2} \frac{\tau}{h^{2}}$, combine the conditions $\frac{1}{6} \leq \lambda \leq \frac{5}{6}, 0<\tau \leq \min \left\{\lambda-\frac{1}{6}, 1-\frac{6}{5} \lambda\right\}$ in the theorem (3.1), we have $\frac{1}{54}<\tau<\frac{1}{13}$. The following figures shows the numerical solution curves and the energy curves for several values of $\tau$ :
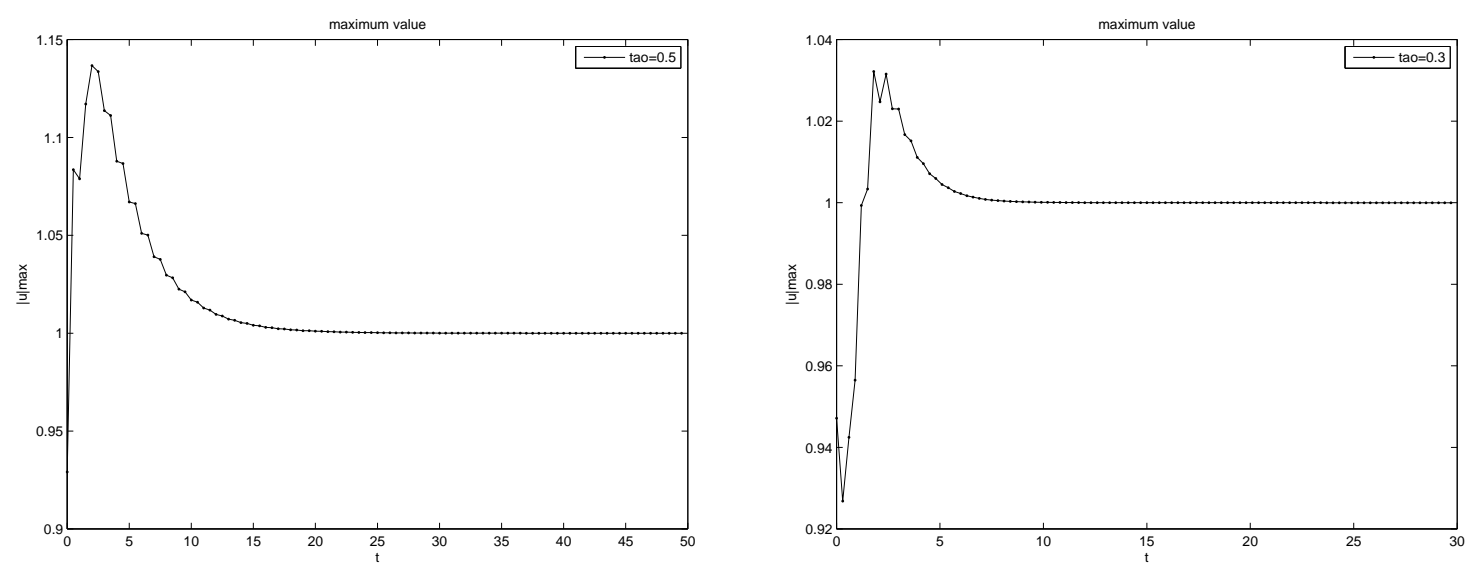

Fig. 1: Maximum values for scheme (2.18) with time step $\tau=0.5,0.3$.
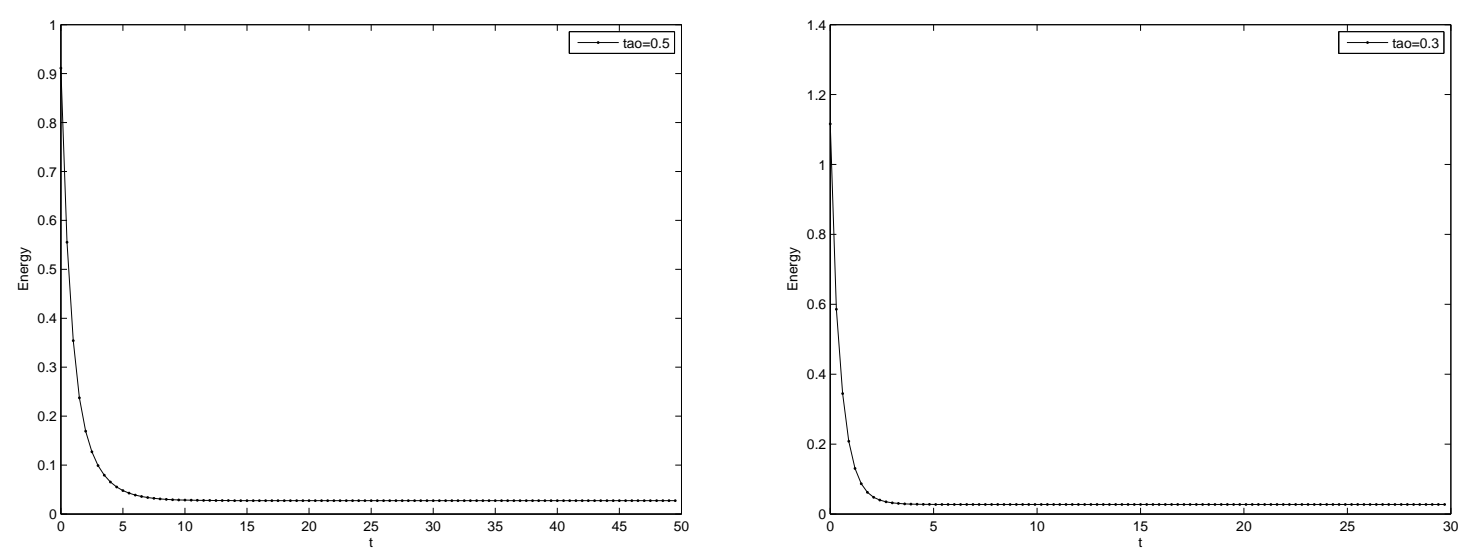

Fig. 2: Energy for scheme (2.18) with time step $\tau=0.5,0.3$. 

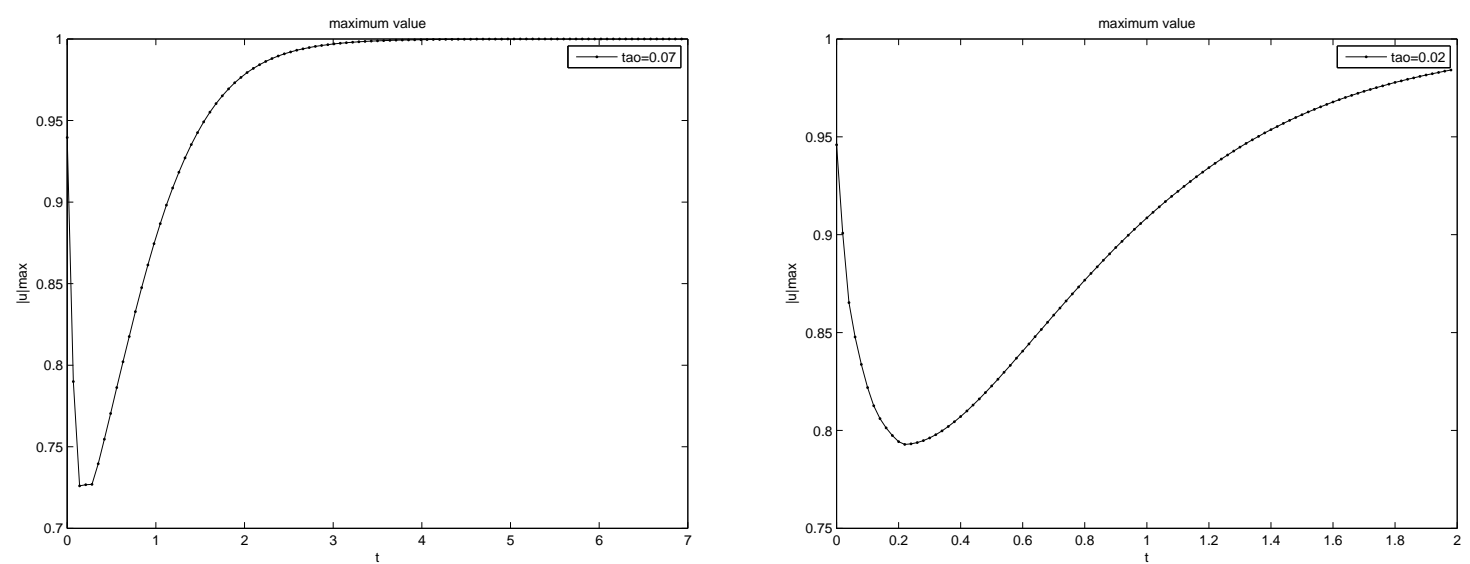

Fig. 3: Maximum values for scheme (2.18) with time step $\tau=0.07,0.02$.
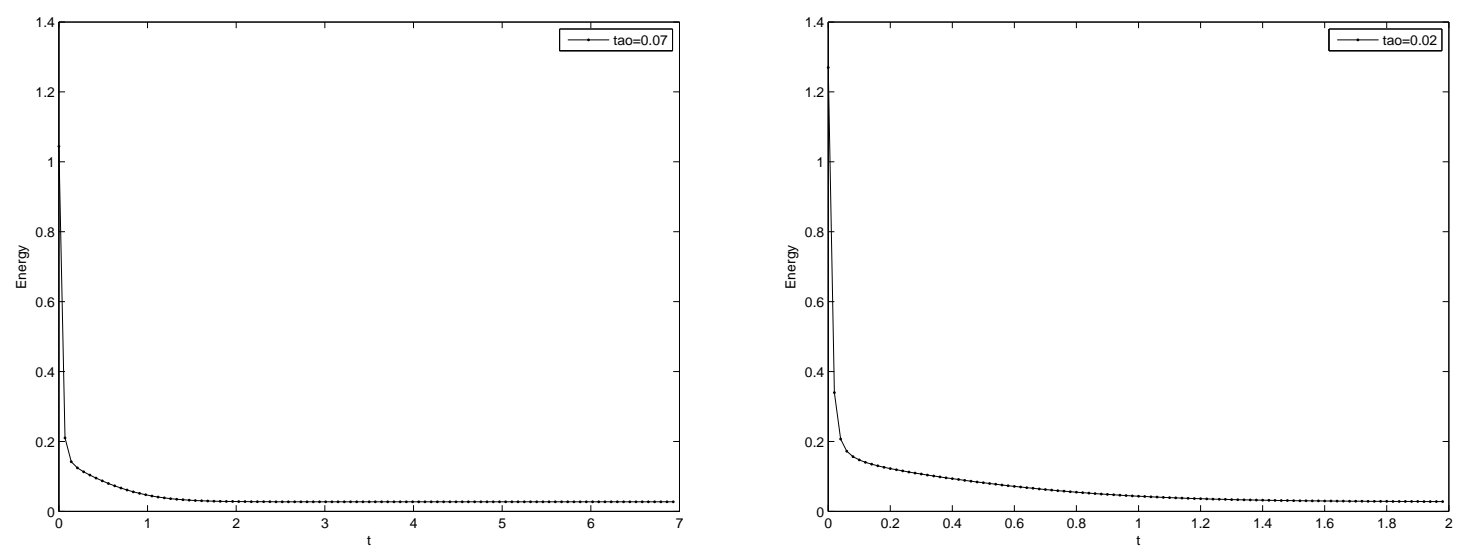

Fig. 4: Energy for scheme (2.18) with time step $\tau=0.07,0.02$.

Fig1 and Fig3 plots the numerical solution of the discrete scheme (2.18) does not satisfy the maximum principle when the time step is $\tau=0.5$ or $\tau=0.3$, but it satisfies the maximum principle when the time step is $\tau=0.07$ and $\tau=0.02$. Moreover, Fig2 and Fig4 plots the discrete energy diminishing stability.

Second, we let the parameter $\epsilon^{2}=0.0001$, and the mesh size in space is $h=0.01$. We have $\frac{1}{6}<\tau<\frac{5}{11}$. When the time step $\tau=0.5$ and $\tau=0.2$, we get the following figures: 

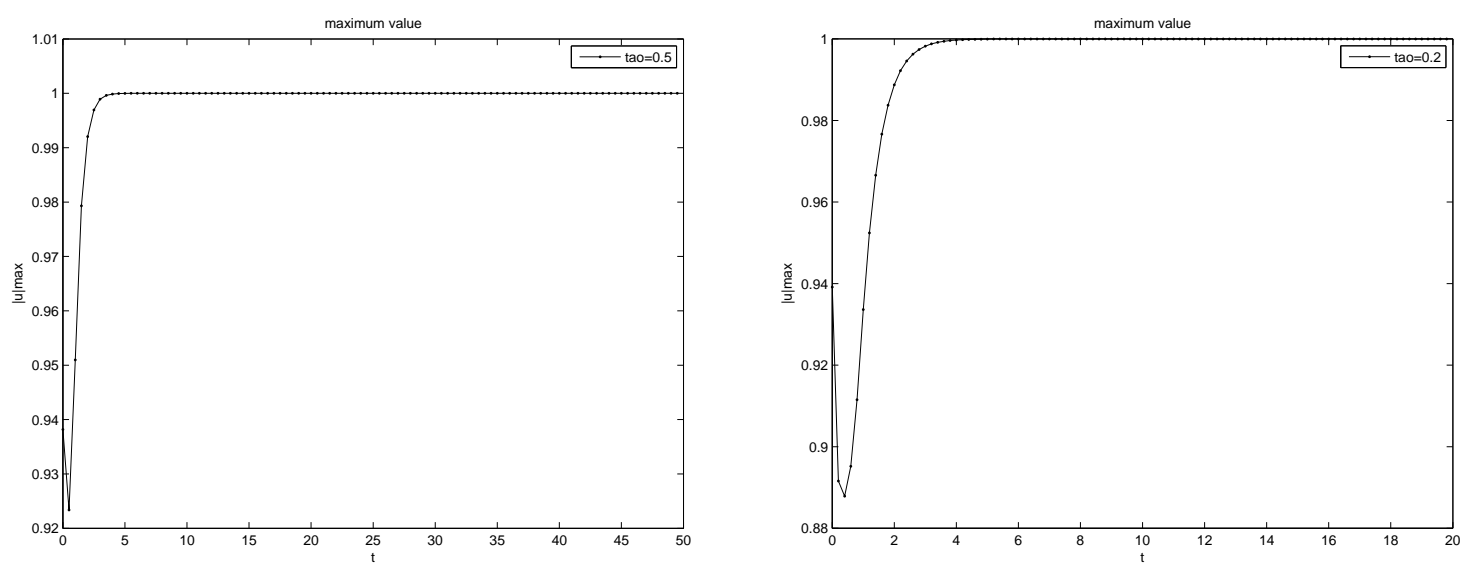

Fig. 5: Maximum values for scheme (2.18) with time step $\tau=0.5,0.2$.
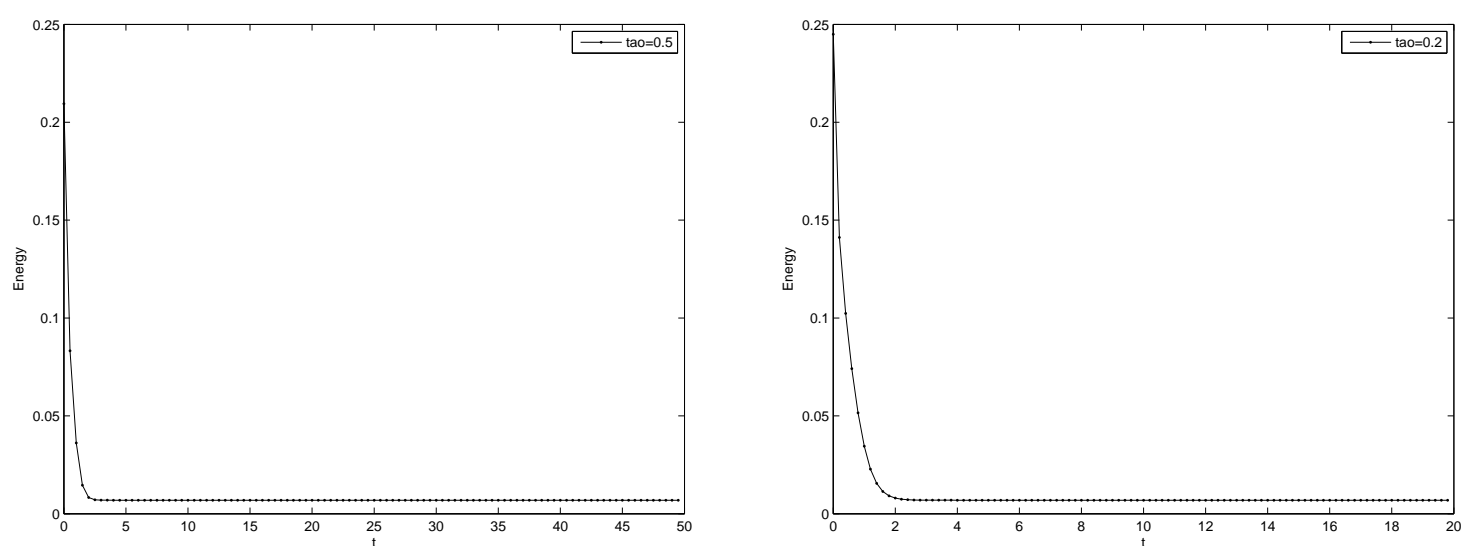

Fig. 6: Energy for scheme (2.18) with time step $\tau=0.5,0.2$.

Fig5 plots the numerical solution of the discrete scheme (2.18) does not satisfy the maximum principle when $\tau=0.5$, but it satisfies the maximum principle when the time step is $\tau=0.2$. Fig6 plots the discrete energy diminishing stability.

\section{Conclusions}

The main contribution of this work is the establishment of the full-discrete fourth-order compact difference scheme for the one-dimensional nonlinear Allen-Cahn equation (2.18). For the fully discretized scheme, we prove that the numerical solution is bounded by the constant 1, i.e. numerical maximum principle. This offers us the possibility to achieve the numerical solution satisfies energy stability. At the same time, the restrictions of the step ratio and the time step $\frac{1}{6} \leq \lambda \leq \frac{5}{6}, 0<$ $\tau \leq \min \left\{\lambda-\frac{1}{6}, 1-\frac{6}{5} \lambda\right\}$ are obtained. Finally, the compact difference scheme is considered as a illustrative example to show the effectiveness and advantages of the proposed scheme.

\section{Acknowledgment}

This work was supported by National Natural Science Foundation of China (No.11761074), the projection of the Department of Science and Technology of JiLin Province (No.20170101052JC) and the Educational Commission of JiLin Province of China (No.JJKH20170453KJ). 


\section{Reference}

[1] Jiaqi Zhang, Tianliang Hou. Discrete Maximum Principle and Energy Stability of Finite Difference Methods for One-dimensional Allen-Cahn Equations [J]. Journal of Beihua University, 2016.4, 17(2):159-164.

[2] Tao Tang, Jiang Yang. Implicit-Explicit Scheme for the Allen-Cahn Equation Preserves the Maximum Principle [J]. Journal of Computational Mathematic, 2016, 34(5):451-461.

[3] Nan Zheng, Shuying Zhai, Zhifeng Weng. Two Efficient Numerical Schemes for the AllenCahn Equation [J]. Advances in Applied Mathematics, 2017, 6(3):283-295.

[4] T.Hou, K.Wang. Discrete Maximum-Norm Stability of A Linearized Second-Order Finite Difference Scheme for Allen-Cahn Equation. Numerical Analysis and Applications, 2017, 10(2):177-183.

[5] Tianliang Hou, Tao Tang, Jiang yang, Numerical Analysis of Fully Discretized Crank-Nicolson Scheme for Fractional-in-Space Allen-Cahn Equations [J]. J Sci Comput,2017, 72:1214-1231.

[6] Tingting Li. Spectral methods for the Allen-Cahn equation and Cahn-Hilliard equation. Huazhong University of Science and Technology, 2015.

[7] Lingling Xu. Second Order Dissipative Difference Scheme for Neumann Boundary Value Problem of Allen-Cahn Equation [D]. Shanghai Jiaotong University, 2010.

[8] Chunli Yu. Local Discontinuous Galerkin Finite Element Method for Allen-Cahn Equation [D]. Shandong University, 2009.

[9] X.B.Feng, A.Prohl. Numerical Analysis of the Allen-Cahn Equation and Approximation for Mean Curvature [J]. Numer Math, 2003, 27(99):33-65.

[10] Jie Shen, Xiaofeng Yang. Numerical Approximations of Allen-Cahn and Cahn-Hilliard Equations [J]. Discrete and Continuous Dynamical Systems, 2010, 28(4):1669-1691.

[11] Yibao Li, Hyun Geun Lee. An Unconditionally Stable Hybrid Numerical Method for Solving the Allen-Cahn Equation [J]. Computers and Mathematics with Applications, 2010, 60:15911606 .

[12] Jeong-Whan Choi, Hyun Geun Lee. An Unconditionally Gradient Stable Numerical Method for Solving the Allen-Cahn Equation [J]. Physica A: Statistical Mechanics and its Applications, 2009, 388:1791-1803. 